\title{
The design and simulation of an optimized MEMS varactor with high $Q$ factor for $R F$ circuits
}

\begin{abstract}
This research concerns on design of high-performance MEMS tuning variable varactor. Varactors can be used as a part of LC-tank of an oscillator. Using MEMS technology causes an improvement in $\mathrm{Q}$ factor of varactor to achieve less phase noise and lower power consumption in an oscillator. In this work a two different gap varactor has been used to avoid the pull-in voltage at $2.4 \mathrm{GHz}$. The expected capacitance is $3 \mathrm{pF}$. The area and the gaps between the plates achieved based on a basic formula of the capacitor. The design of layout has been done by CoventorWare software 2006. The DC voltage is $2.5 \mathrm{~V}$ which is applied to the plates and the results of $3.02 \mathrm{pF}$ could be gained after analyzing. The $\mathrm{Q}$ factor of varactor calculated based on a distributed RC line and computed about 376.43 using MATLAB programming. The result is good enough to make low phase noise in VCO.
\end{abstract}

